\section{In silico gene selection strategy for custom microarray design}

\author{
Vijay R. Dondeti, Conor W. Sipe, and Margaret S. Saha \\ College of William and Mary, Williamsburg, VA, USA
}

BioTechniques 37:768-776 (November 2004)

Microarray technology has become an important tool for studying large-scale gene expression for a diversity of biological applications. However, there are a number of experimental settings for which commercial arrays are either unsuitable or unavailable despite the existence of sequence information. With the increasing availability of custom array manufacturing services, it is now feasible to design high-density arrays for any organism having sequence data. However, there have been relatively few reports discussing gene selection, an important first step in array design. Here we propose an in silico strategy for custom microarray gene selection that is applicable to a wide range of organisms, based on utilizing public domain microarray information to interrogate existing sequence data and to identify a set of homologous genes in any organism of interest. We demonstrate the utility of this approach by applying it to the selection of candidate genes for a custom Xenopus laevis microarray. A significant finding of this study is that 3\%-4\% of Xenopus expressed sequence tags (ESTs) are in an orientation contrary to that indicated in the public database entry (http://mssaha. people.wm.edu/suppMSS.htm,).

\section{INTRODUCTION}

Microarray technology has become a powerful and increasingly common tool for high-throughput analysis of gene expression in a wide variety of biological fields. While commercial arrays are readily available for several important species, there are a number of organisms and model systems for which arrays do not exist, despite the availability of considerable sequence data. Even for organisms that have commercial arrays readily available, specialized arrays may be more suitable for a given experiment. With many companies offering custom probe design and array manufacturing services, it has now become feasible for investigators to design high-density microarrays for specific applications. For many organisms, current chip densities do not generally permit the use of all available sequences for a given genome (1); moreover, the use of multiple chips is economically prohibitive and introduces greater experimental variability. On the other hand, the probe density of current arrays is sufficiently large to make a manual selection of the genes impractical at best. To date, the technical aspects of probe design have been the focus of considerable discussion $(2,3)$, however, the selection of the genes included on an array $(4,5)$, a critical first step in a custom-designed microarray experiment, has received less attention.

The majority of papers reporting custom-designed arrays utilize a cDNA approach that relies on randomly generated expressed sequence tag (EST) libraries to dictate the number and makeup of probe sequences included on a chip $(6,7)$. Due to the effort and expense associated with constructing custom cDNA arrays, the use of long oligonucleotide arrays has become increasingly popular as an alternative platform (8-10). Experimental evidence has validated their efficacy, and it has been suggested that oligonucleotide microarrays are more reliable for determining changes in gene expression than their cDNA counterparts (11).

The increasing availability of custom-designed high-density arrays has presented investigators with the daunting task of selecting a large number of genes for inclusion on such few systematic methods presented in the literature to aid in the process of gene selection. Lorenz et al. (13) propose a strategy for the design of a an array (12). To date, there have been mouse immunology array that involves selecting Unigene clusters based on their expression in immunological tissues. A ranked list of these clusters is generated based on the relevance of the library origin, and a representative sequence from each cluster is chosen for inclusion on the array. While this method is applicable to the design of specialized arrays, it is difficult to apply to the selection of genes for more general arrays; that is, those designed to investigate processes involving genes from many tissue types or when working without a priori knowledge of a target. Band et al. (14) designed a bovine array using successive Basic Local Alignment Search Tool (BLAST) searches (http://www.ncbi.nlm.nih. gov/BLAST/) against the human Unigene database to cull putative orthologs and novel organism-specific genes from a large pool of ESTs for inclusion on a cDNA array. Another tool useful in the design of microarrays for a select set of model organisms is Onto-Design, which allows users to choose sequences based on their gene ontology (GO) classification (15). This permits the design of arrays focused on specific functional pathways or biological processes. A related tool, Onto-Compare, gives investigators the ability to perform a functional analysis of numerous commercial chips as well as user-defined custom arrays $(15,16)$. Such an analysis provides a better understanding of the pathways and processes that can be investigated with a given microarray.

Considerable effort has already been expended on generating EST libraries for the design of custom arrays to study specific biological processes. Given that the details of these arrays are publicly available, we set out to devise an in silico method for the design of custom microarrays that exploits this growing body of information and is applicable to any organism having sequence data available. This gene selection protocol is applicable to the design of both general and more specialized array types and is most suitable for designing microarrays to investigate processes that are conserved among species. In the current report, we demonstrate the utility of this technique by applying it to the design of a custom 
high-density oligonucleotide microarray for the organism Xenopus laevis $(17,18)$. Significantly, these results reveal that one cannot rely on sequence submission conventions to determine proper sequence orientation (i.e., sense or antisense).

\section{MATERIALS AND METHODS}

A set of input sequences was derived from 60 publicly available human microarrays that represented both general and functionally specialized subsets of the genome (Table 1). The information for arrays 1-42 was taken from SuperArray (Frederick, MD, USA) and for arrays 46-59 from BD Biosciences Clontech (Palo Alto, CA, USA). The detailed source information for arrays 43-45 and 60 is noted in Table 1.

Using this information, a text file for each array was prepared containing the accession numbers of each gene represented on the array, following the removal of the sequences used as hybridization controls. These files were individually submitted to the Batch Entrez tool, and the corresponding sequences were retrieved in FASTA format directly from the GenBank ${ }^{\circledR}$ database on December 8, 2003. Each of these sequence files was aligned against the Xenopus Unigene unique database (Xl.seq.uniq, Build 48 containing 21,810 clusters) using a local BLAST server installed on a desktop PC. Blastn search parameters were set such that no more than one sequence (the best-hit) was returned for each query sequence. Using the array information, another text file (hereafter referred to as the description file) containing the accession numbers and corresponding descriptions was prepared for each input array. Thus, for each array, two associated files were generated, the BLAST results file and the description file.

The two files corresponding to each array were submitted as input to a Perl script in the order shown in Table 1. The script, which utilizes portions of the Bioperl toolkit (19), scans the BLAST results file and isolates both the source and Xenopus accession numbers, FASTA definition lines, expectation (E)-value, and orientation of the resulting alignment. In addition, the script also retrieves the Unigene cluster ID and the Unigene sequence identifier (SID) corresponding to the Xenopus accession number. If an alignment passed the set threshold (E $<1$ e-15), these values, along with the corresponding description retrieved from the description file, were stored in a Microsoft ${ }^{\circledR}$ Access database in the appropriate fields (Table 2). If the Xenopus sequence was already contained in the database, only the comment field was updated, indicating the sequence was found on multiple input arrays. The Onto-Compare tool was used to evaluate the functional aspects of the selected gene set (16).

To determine the proper orientation of the selected Xenopus sequences, we used the features listed in each Unigene record. With the aid of a second Perl script, the corresponding Unigene records were downloaded based on the SID, and the required features were retrieved and entered into the database. The script then checked for consistency of the features, and the results of the analysis were entered into the database. A few sequences had features that were inconsistent with regard to orientation, and given that they comprised a small fraction of the data set, were not taken into further consideration. The second Perl script was modified to perform the same analysis on the entire Xenopus Unigene unique database. Highthroughput cDNA (HTC) sequences and ESTs with no read information (i.e., $5^{\prime}$ or $3^{\prime}$ ) amount to $<0.1 \%$ of all Xenopus sequences and were not considered when determining error rates because there is no apparent convention for submission with respect to orientation.

Sequences in the selected set that did not have any Unigene features were aligned against the GenBank nucleotide database (downloaded on January 12, 2004) using a local BLAST server and analyzed as described below. Blastn search parameters were set such that only alignments having an $\mathrm{E}<1 \mathrm{e}-6$ were returned. All Perl scripts and supplementary materials are available online at http://mssaha.people.wm.edu/ suppMSS.html. 
Table 1. Summary Statistics for Each Source Array

\begin{tabular}{|c|c|c|c|c|}
\hline Number & Array Title & $\begin{array}{l}\text { Total Input } \\
\text { Sequences }\end{array}$ & $\begin{array}{c}\text { Passed } \\
\text { Thresholda }^{a}\end{array}$ & $\begin{array}{l}\text { Nonredundant } \\
\text { Xenopus Hits }\end{array}$ \\
\hline 1 & Human Androgen Signaling and Prostate Cancer Gene Array & 100 & 39 & 39 \\
\hline 2 & Human Angiogenesis Gene Array & 96 & 31 & 28 \\
\hline 3 & Human Apoptosis Gene Array & 96 & 5 & 5 \\
\hline 4 & Human Asthma Gene Array & 85 & 4 & 3 \\
\hline 5 & Human Breast Cancer and Estrogen Signaling Pathway Gene Array & 96 & 36 & 22 \\
\hline 6 & Human cAMP/Ca2+ PathwayFinder Gene Array & 96 & 39 & 31 \\
\hline 7 & Human Cancer Drug Resistance \& Metabolism Gene Array & 96 & 35 & 21 \\
\hline 8 & Human Cancer PathwayFinder Gene Array & 96 & 33 & 11 \\
\hline 9 & Human Cancer PathwayFinder II Gene Array & 96 & 30 & 13 \\
\hline 10 & Human Na2+/NF-AT Signaling Pathways Gene Array & 96 & 42 & 25 \\
\hline 11 & Human Cardiovascular Disease Gene Array I: Biomarkers & 96 & 11 & 5 \\
\hline 12 & Human Cardiovascular Disease Gene Array II: Atherosclerosis Gene Array & 96 & 22 & 8 \\
\hline 13 & Human Cell Cycle Gene Array & 96 & 46 & 36 \\
\hline 14 & Human Chemokine and Receptor Gene Array & 77 & 3 & 2 \\
\hline 15 & Human Common Cytokine Gene Array & 96 & 15 & 6 \\
\hline 16 & Human DNA Damage Signaling Pathways Gene Array & 96 & 32 & 20 \\
\hline 17 & Human Drug Metabolism Gene Array & 95 & 10 & 6 \\
\hline 18 & Human Extracellular Matrix \& Adhesion Molecules Gene Array & 96 & 22 & 6 \\
\hline 19 & Human EGF/PDGF Signaling Pathway Gene Array & 96 & 63 & 27 \\
\hline 20 & Human Endothelial Cell Biology Gene Array & 96 & 14 & 2 \\
\hline 21 & Human Genome Stability/DNA Repair Gene Array & 96 & 25 & 14 \\
\hline 22 & Human Glucocorticoid Signaling I Gene Array & 96 & 24 & 18 \\
\hline 23 & Human G Protein-coupled Receptors Signaling PathwayFinder Gene Array & 96 & 22 & 7 \\
\hline 24 & Human Growth Factor Gene Array & 96 & 17 & 3 \\
\hline 25 & Human Hypoxia Signaling Pathway Gene Array & 96 & 47 & 33 \\
\hline 26 & Human Inflammatory Cytokine/Receptor Gene Array & 96 & 3 & 0 \\
\hline 27 & Human Insulin Signaling Pathway Gene Array & 96 & 55 & 23 \\
\hline 28 & Human Interleukin and Receptor Gene Array & 72 & 1 & 0 \\
\hline 29 & Human JAK/STAT Signaling Pathway Gene Array & 95 & 29 & 12 \\
\hline 30 & Human MAP Kinase Signaling Pathways Gene Array & 96 & 57 & 10 \\
\hline 31 & Human Neuroscience-1 Ion Channel \& Transporter Gene Array & 96 & 43 & 32 \\
\hline 32 & Human Neurotrophin and Receptors Gene Array & 96 & 31 & 9 \\
\hline 33 & Human NFkB Signaling Pathway Gene Array & 96 & 21 & 3 \\
\hline 34 & Human Nitric Oxide Gene Array & 96 & 25 & 8 \\
\hline 35 & Human p53 Signaling Pathway Gene Array & 96 & 41 & 12 \\
\hline 36 & Human Signal Transduction PathwayFinder Gene Array & 96 & 25 & 2 \\
\hline 37 & Human Stem Cell Gene Array & 262 & 112 & 51 \\
\hline 38 & Human Stress \& Toxicity PathwayFinder Gene Array & 96 & 27 & 8 \\
\hline 39 & Human TGF-B BMP Signaling Pathway Gene Array & 96 & 47 & 12 \\
\hline 40 & Human Th1-Th2-Th3 Gene Array & 96 & 19 & 4 \\
\hline 41 & Human Tumor Metastasis Gene Array & 96 & 31 & 2 \\
\hline 42 & Human Wnt Signaling Pathway Gene Array & 96 & 56 & 24 \\
\hline 43 & Barrett et al. (23) & 1093 & 91 & 58 \\
\hline 44 & Hata et al. (24) & 40 & 8 & 6 \\
\hline 45 & Signal Transduction Array (http://www.grc.nia.nih.gov/branches/rrb/dna/array.htm) & 708 & 67 & 17 \\
\hline 46 & Human Apoptosis Array & 214 & 77 & 17 \\
\hline 47 & Human Cancer 1.2 Array & 1183 & 446 & 165 \\
\hline
\end{tabular}


Table 1. Summary Statistics for Each Source Array (continued)

\begin{tabular}{|c|c|c|c|c|}
\hline Number & Array Title & $\begin{array}{l}\text { Total Input } \\
\text { Sequences }\end{array}$ & $\begin{array}{c}\text { Passed } \\
\text { Thresholda }^{\text {Pa }}\end{array}$ & $\begin{array}{l}\text { Nonredundant } \\
\text { Xenopus Hits }^{\mathrm{b}}\end{array}$ \\
\hline 48 & Human Cardiovascular Array & 607 & 173 & 61 \\
\hline 49 & Human Cell Cycle Array & 120 & 71 & 0 \\
\hline 50 & Human Cell Interaction Array & 273 & 91 & 4 \\
\hline 51 & Human Cytokine/Receptor Array & 276 & 85 & 3 \\
\hline 52 & Human Hematology/Immunology Array & 413 & 121 & 47 \\
\hline 53 & Human Neurobiology Array & 597 & 264 & 101 \\
\hline 54 & Human Oncogene Array & 548 & 230 & 81 \\
\hline 55 & Human Stress Array & 243 & 97 & 14 \\
\hline 56 & Human Toxicology 1.2 Array & 1185 & 492 & 96 \\
\hline 57 & Human Tumor Array & 437 & 118 & 30 \\
\hline 58 & Human Oncogene/Tumor Suppressor Array & 199 & 96 & 4 \\
\hline 59 & Clontech $13 \mathrm{~K}$ Human Oligos for custom array design & 12716 & 3353 & 1643 \\
\hline \multirow[t]{2}{*}{60} & Hsaio et al. (25) & 6561 & 2059 & 79 \\
\hline & TOTAL & 31559 & 9229 & 3029 \\
\hline \multicolumn{5}{|c|}{$\begin{array}{l}\text { The leftmost column indicates the order in which the source arrays were processed. Gene lists for arrays 1-42 are available at www.superarray.com/ArrayList.php; } \\
\text { see www.bdbiosciences.com/clontech/atlas/genelists/index.shtml for arrays } 46-59 \text {. } \\
\text { aNumber of Xenopus sequences passing the threshold expectation (E)-value. } \\
\text { bNumber of nonredundant Xenopus sequences added to the candidate database. }\end{array}$} \\
\hline
\end{tabular}

Table 2. Structure of Candidate Gene Database

\begin{tabular}{|c|c|}
\hline Field & Description \\
\hline SourceAccession & Accession number of the source gene. \\
\hline SourceDefinitionLine & $\begin{array}{l}\text { Definition line obtained from FASTA sequence of the source } \\
\text { gene. }\end{array}$ \\
\hline SourceDescription & Description of the source gene provided by array source. \\
\hline Accession & Accession number of the homologous Xenopus gene. \\
\hline ClusterID & Unigene cluster ID corresponding to the Xenopus gene. \\
\hline SID & Unigene sequence identifier corresponding to the Xenopus gene. \\
\hline DefinitionLine & $\begin{array}{l}\text { Definition line obtained from the Unigene entry of the Xenopus } \\
\text { gene. }\end{array}$ \\
\hline Comment & $\begin{array}{l}\text { Used to track the source microarray from which the sequence } \\
\text { was obtained. }\end{array}$ \\
\hline EValue & E-value resulting from the BLAST search. \\
\hline Plus/Minus & Orientation of the alignment from the resulting BLAST report. \\
\hline
\end{tabular}

\section{RESULTS AND DISCUSSION}

Sixty human microarrays were chosen as a starting point for the development of the technique because their gene lists were both freely available and in a format that could be easily manipulated. After downloading the sequences represented on these microarrays from the GenBank database, each sequence in this set was aligned to the $X$. laevis Unigene unique database, which is composed of a representative sequence from each cluster having the longest region of high-quality sequence data. These BLAST results were parsed using a Perl script, and sequences homologous to input source sequences were selected based on a user-defined threshold value of $\mathrm{E}<1 \mathrm{e}-15$. Band et al. (20) used a less stringent threshold value of $\mathrm{E}<1 \mathrm{e}-5$ when comparing two mammalian species. However, given that our comparison was between more distantly related organisms, namely amphibian and mammalian species, a more stringent threshold value was chosen to ensure that we obtained biologically meaningful homologous Xenopus genes (1).

Of the 31,599 human sequences that were derived from the 60 arrays and aligned against the $X$. laevis Unigene unique database, 9229 sequences passed the set $\mathrm{E}<1 \mathrm{e}-15$ threshold. After the removal of duplicate gene entries by excluding sequences with an accession number already present in the set, 3029 nonredundant candidate $X$. laevis genes remained (Table 1 and the Supplementary Table 1 available at http://mssaha.people.wm.edu/ suppMSS.html). This set of candidate genes was stored in a database, the structure of which is given in Table 2 . The final number of candidate sequences obtained by this method will vary depending on the stringency of the E-value applied in the selection criteria. The use of a less stringent threshold value will increase the number of sequences selected, although confidence in the homology of the resulting sequences would diminish. In order to evaluate the functional pathways and biological processes encompassed by the selected gene set, the corresponding source genes were submitted to OntoCompare (16). This tool is complementary to the gene selection strategy described here, as the results of such an analysis can reveal underrepresented functional categories. It is evident from the Onto-Compare output that the selected Xenopus sequence set covers a broad set of functions and processes (Figure 1). 
Table 3. Percentages of Sequences Determined To Be in an Orientation Contrary to That Indicated in the Database

\begin{tabular}{|c|c|c|c|c|c|}
\hline & \multicolumn{3}{|c|}{ Selected Xenopus Sequence Set } & \multicolumn{2}{|c|}{ Xenopus Unigene Unique Database } \\
\hline & $>1$ Feature & $\geq 1$ Feature & Alla & $>1$ Feature & $\geq 1$ Feature \\
\hline 3' EST & $2 / 106(1.9 \%)$ & $2 / 144(1.4 \%)$ & $4 / 191(2.1 \%)$ & 28/912 (3.1\%) & $42 / 1348(3.1 \%)$ \\
\hline $5^{\prime}$ EST & $35 / 634(5.5 \%)$ & $48 / 780(6.2 \%)$ & $76 / 1077(7.0 \%)$ & $98 / 2438(4.0 \%)$ & $138 / 3333(4.1 \%)$ \\
\hline mRNA & $0 / 1466(0 \%)$ & $1 / 1700(0.06 \%)$ & $1 / 1705(0.06 \%)$ & $1 / 3156(0.03 \%)$ & $2 / 3722(0.05 \%)$ \\
\hline
\end{tabular}

Given that ESTs make up a large percentage of the available sequences for many organisms, and in light of the reported incidences of inverted clones in EST libraries $(7,21)$, it was important to determine the correct orientation of the selected sequences. Therefore, we utilized data contained in the Unigene records that provide the location and orientation (plus/minus strand) of the coding sequence (CDS) and protein similarity (prot_sim) features. The protein similarities were derived from the NCBI ProtEST database (http:// www.ncbi.nlm.nih.gov/UniGene/ ProtEST/), and the method considered only human, mouse, and rat alignments.

Of the 3029 selected Xenopus sequences, the Unigene records of $2667 \mathrm{had}$ at least one feature (CDS and/or prot_sim). We determined sequence orientation by checking for consistency among the feature types for a given record. In the cases where Unigene records had two or more features present $(n=2244)$, an orientation was assigned only if all of the features agreed on direction $(n=2241)$. There were only three sequences that had two or more features that were inconsistent with one another in regard to orientation. If only one feature was available $(n=423)$, its orientation was taken to be the correct orientation for the sequence.

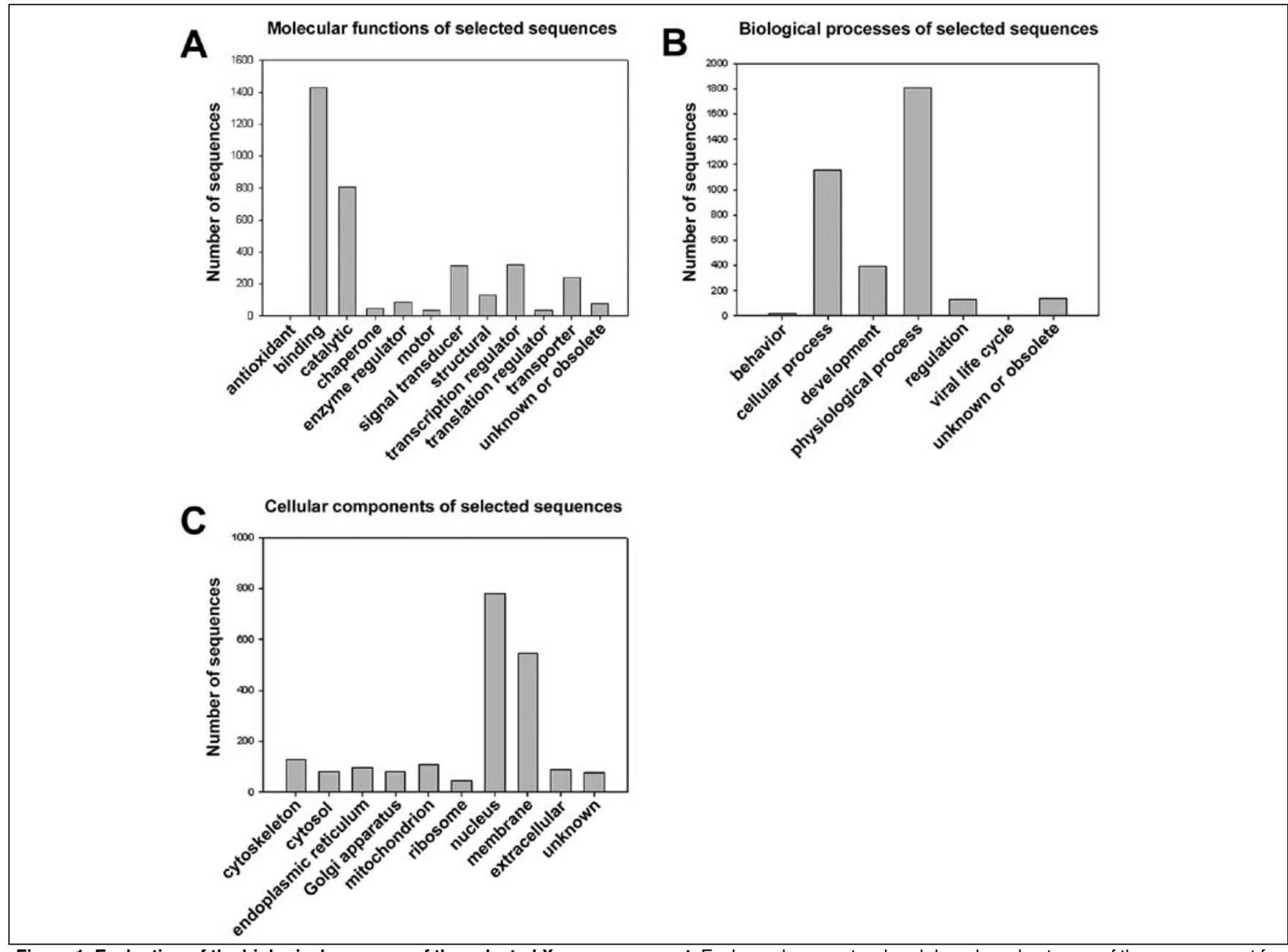

Figure 1. Evaluation of the biological coverage of the selected Xenopus gene set. Each graph presents a breakdown by subcategory of the sequence set for the three main divisions of the gene ontology (GO) hierarchy: (A) molecular function, (B) biological process, and (C) cellular component. 
GenBank sequence submission convention dictates that mRNA and $5^{\prime}$ EST sequences should be submitted in the sense orientation, and 3' ESTs in the antisense orientation (R.C. Morris, NCBI, personal communication). Using the above Xenopus sequences for which proper orientation could be ascertained, we set out to determine the degree to which this convention could be relied upon for determining sequence orientation (Table 3). To obtain a more accurate estimation of the percentage of EST and mRNA sequences annotated contrary to standard convention, this procedure was applied to the entire Xenopus Unigene unique database $(n=21,810)$. Of this number, 1909 sequences had a single Unigene feature present, and 6594 had more than one feature. For the sequences that had more than one feature, 6573 were consistent with respect to orientation, whereas 21 sequences were not. The error rates in reported orientation for this data set are shown in Table 3. These data demonstrate that approximately 3\%-4\% of the EST sequences in the database are annotated incorrectly with respect to orientation, whereas mRNA sequences have an extremely low error rate $(0.05 \%)$ (see Supplementary Table 2 at http:/ mssaha.people.wm.edu/suppMSS.html for a list of all erroneously annotated sequences). This may actually be an underestimate of the error rates in the Xenopus Unigene database as a whole, given that this method was applied to a representative sequence having the longest region of high-quality sequence data from each of the 21,810 clusters as opposed to the entire Xenopus Unigene database.

The orientation of 362 sequences in the selected set could not be determined due to a lack of any features in their Unigene records. However, given the low incidence of incorrect annotation for mRNAs, we were able to determine with confidence the orientation for 351 of these sequences by aligning them against the GenBank nucleotide database (containing no ESTs) using Blastn on a local server and inspecting the search results for a consensus of homologous sequences from other organisms. The search results for the remaining 11 sequences were inconclusive and, therefore, were eliminated from further consideration. Taking these 351 sequences into account, we calculated an overall estimation for the rate of incorrect assignment of orientation in the selected Xenopus sequence set (Table 3, see the "All" column). Errors in the assignment of orientation are most likely the result of human oversight (i.e., mislabeling, contamination, etc.) or cloning artifacts (shown by the presence of repetitive elements, vector, linker, or primer in several of the incorrectly annotated sequences).

Due to the high-throughput nature of microarray experiments, the error rates in the orientation of sequences revealed by this study have the potential to affect the reliability of microarray studies, particularly for oligonucleotide arrays, as the probes spotted on a chip are single-stranded. If the incorrect strand for a gene is used as the probe, no signal 
would be present even if the gene was expressed at the experimental conditions under investigation. In contrast, a lack of knowledge concerning correct orientation does not pose a comparable problem for cDNA-based arrays given that the probes are double-stranded. Nonetheless, without proper knowledge of sequence orientation, any complete validation of the microarray results would remain problematic.

In this study, we have presented an in silico strategy for the selection of genes for inclusion on a microarray that is based on interrogating publicly available microarray information and the GenBank database. This technique can be applied to the design of microarrays for any organism of interest that has sequence data available and can be tailored to a given chip density. The power and utility of this selection method will increase as the body of available microarray information expands. This work also demonstrates that it is essential to examine closely the orientation of sequences selected for inclusion on an oligonucleotide microarray to ensure the reliability of microarray data. This study adds to a growing number of issues encountered when relying on public databases for microarray gene selection $(4,22)$.

\section{ACKNOWLEDGMENTS}

The authors wish to thank Ken Cho for kindly providing his microarray expertise and sharing unpublished material. We also thank Michael Trosset for helpful discussions. In addition, we would like to thank Lewis Geer, Lukas Wagner, and other members of the NCBI staff. This work was supported in part by the Commonwealth Technology Research Fund of Virginia, a Howard Hughes Medical Institute Biological Sciences Undergraduate Education Program Grant to the College of William and Mary, NSF IBN-9553176 to M.S.S., and a Beckman Scholars award to V.R.D.

\section{COMPETING INTERESTS STATEMENT}

The authors declare no competing interests.

\section{REFERENCES}

1.Smith, J., D. Speed, A.S. Law, E.J. Glass, and D.W. Burt. 2004. In-silico identification of chicken immune-related genes. Immunogenetics 56:122-133.

2.Rouillard, J.-M., M. Zuker, and E. Gulari. 2003. OligoArray 2.0: design of oligonucleotide probes for DNA microarrays using a thermodynamic approach. Nucleic Acids. Res. 31:3057-3062.

3.Nielsen, H.B., R. Wernersson, and S. Knudsen. 2003. Design of oligonucleotides for microarrays and perspectives for design of multi-transcriptome arrays. Nucleic Acids Res. 31:3491-3496.

4.Lambert, J.C., E. Testa, V. Cognat, J. Soula, D. Hot, Y. Lemoine, G. Gaypay, and P. Amouyel. 2003. Relevance and limitations of public databases for microarray design: a critical approach to gene predictions. Pharmacogenomics J. 3:235-241.

5.Edgerton, M.E., R. Taylor, J.I. Powell, L. Hunter, R. Simon, and E.T. Liu. 2002. A bioinformatics tool to select sequences for microarray studies of mouse models of oncogenesis. Bioinformatics 18:774-775.

6.Cogburn, L.A., X. Wang, W. Carre, L. Rejto, T.E. Porter, S.E. Aggrey, and J. Simon. 2003. Systems-wide chicken DNA microarrays, gene expression profiling, and discovery of functional genes. Poult. Sci. 82:939-951.

7.Whitfield, C.W., M.R. Band, M.F. Bonaldo, C.G. Kumar, L. Liu, J.R. Pardinas, H.M. Robertson, M.B. Soares, et al. 2002. Annotated expressed sequence tags and cDNA microarrays for studies of brain and behavior in the honey bee. Genome Res. 12:555-566.

8.Barczak, A., M.W. Rodriguez, K. Hanspers, L.L. Koth, Y.C. Tai, B.M. Bolstad, T.P. Speed, and D.J. Erle. 2003. Spotted long oligonucleotide arrays for human gene expression analysis. Genome Res. 13:1775-1785.

9.Kuo, W.P., T.K. Jenssen, A.J. Butte, L. Ohno-Machado, and I.S. Kohane. 2002. Analysis of matched mRNA measurements from two different microarray technologies. Bioinformatics 18:405-412.

10.Tan, P.K., T.J. Downey, E.L. Spitznagel Jr., P. Xu, D. Fu, D.S. Dimitrov, R.A. Lempicki, B.M. Raaka, et al. 2003. Evaluation of gene expression measurements from commercial microarray platforms. Nucleic Acids Res. 31:5676-5684.

11.Li, J., M. Pankratz, and J.A. Johnson. 2002. Differential gene expression patterns revealed by oligonucleotide versus long cDNA arrays. Toxicol. Sci. 69:383-390.

12.Wurmbach, E., T. Yuen, and S.C. Sealfon. 2003. Focused microarray analysis. Methods 31:306-316.

13.Lorenz, M.G., L.M. Cortes, J.J. Lorenz, and E.T. Liu. 2003. Strategy for the design of custom cDNA microarrays. BioTechniques 34:1264-1270.

14.Band, M.R., C. Olmstead, R.E. Everts, Z.L. Liu, and H.A. Lewin. 2002. A 3800 gene microarray for cattle functional genomics: comparison of gene expression in spleen, placenta, and brain. Anim. Biotechnol. 13:163-172.

15.Draghici, S., P. Khatri, P. Bhavsar, A. Shah, S.A. Krawetz, and M.A. Tainsky. 2003.
Onto-Tools, the toolkit of the modern biologist: Onto-Express, Onto-Compare, Onto-Design and Onto-Translate. Nucleic Acids Res. 31:3775-3781.

16.Draghici, S., P. Khatri, A. Shah, and M.A. Tainsky. 2003. Assessing the functional bias of commercial microarrays using the ontocompare database. BioTechniques Suppl:5561.

17.Altmann, C.R., E. Bell, A. Sczyrba, J. Pun, S. Bekiranov, T. Gaasterland, and A.H. Brivanlou. 2001. Microarray-based analysis of early development in Xenopus laevis. Dev. Biol. 236:64-75.

18.Munoz-Sanjuan, I., E. Bell, C.R. Altmann, A. Vonica, and A.H. Brivanlou. 2002. Gene profiling during neural induction in Xenopus laevis: regulation of BMP signaling by post-transcriptional mechanisms and TAB3, a novel TAK1-binding protein. Development 129:5529-5540.

19.Stajich, J.E., D. Block, K. Boulez, S.E. Brenner, S.A. Chervitz, C. Dagdigian, G. Fuellen, J.G. Gilbert, et al. 2002. The Bioperl toolkit: Perl modules for the life sciences. Genome Res. 12:1611-1618.

20.Band, M.R., J.H. Larson, M. Rebeiz, C.A. Green, D.W. Heyen, J. Donovan, R. Windish, C. Steining, et al. 2000. An ordered comparative map of the cattle and human genomes. Genome Res. 10:1359-1368.

21.Hillier, L.D., G. Lennon, M. Becker, M.F. Bonaldo, B. Chiapelli, S. Chissoe, N. Dietrich, T. DuBuque, et al. 1996. Generation and analysis of 280,000 human expressed sequence tags. Genome Res. 6:807-828.

22.Mecham, B.H., G.T. Klus, J. Strovel, M. Augustus, D. Byrne, P. Bozso, D.Z. Wetmore, T.J. Mariani, et al. 2004. Sequence-matched probes produce increased cross-platform consistency and more reproducible biological results in microarray-based gene expression measurements. Nucleic Acids Res. 32:e74.

23.Barrett, T., C. Cheadle, W.B. Wood, D. Teichberg, D.M. Donovan, W.J. Freed, K.G. Becker, and M.P. Vawter. 2001. Assembly and use of a broadly applicable neural cDNA microarray. Restor. Neurol. Neurosci. 18:127135.

24.Hata, R., M. Masumura, H. Akatsu, F. Li, H. Fujita, Y. Nagai, T. Yamamoto, H. Okada, et al. 2001. Up-regulation of calcineurin Abeta mRNA in the Alzheimer's disease brain: assessment by cDNA microarray. Biochem. Biophys. Res. Commun. 284:310-316.

25.Hsiao, L.L., F. Dangond, T. Yoshida, R. Hong, R.V. Jensen, J. Misra, W. Dillon, K.F. Lee, et al. 2001. A compendium of gene expression in normal human tissues. Physiol. Genomics 7:97-104.

Received 3 June 2004; accepted 6 July 2004.

Address correspondence to Margaret $S$. Saha, Department of Biology, College of William and Mary, P.O. Box 8795, Williamsburg,VA 23187,USA.e-mail: mssaha@wm. $e d u$ 